\title{
Nephritis Associated with Ulcerative Colitis
}

\author{
Hirobumi Tokuyama, Shu Wakino, Koichi Hayashi and Hiroshi Itoh \\ Department of Internal Medicine, School of Medicine, Keio University, Tokyo, \\ Japan
}

\section{Introduction}

Ulcerative colitis (UC) is an idiopathic chronic inflammatory disease of the colon and rectum, characterized by mucosal inflammation and typically presenting with bloody diarrhea. Crohn's disease is characterized by transmural inflammation of the gut wall and can affect any part of the tubular gastrointestinal tract. Although the underlying etiology and exact pathogenesis remain fully unclarified, current hypothesis favors dysregulation of gastrointestinal immune system in genetically predisposed individuals [1]. Extra-intestinal manifestations of inflammatory bowel disease (IBD) are common, ensuing in approximately $40 \%$ of patients [2], many of which are postulated to be associated with autoimmune mechanisms [3]. Renal manifestations associated with IBD, however, have rarely been reported. Sulfasalazine reaches the colon intact, where it is metabolized to 5-aminosalicylic acid (5-ASA, mesalazine, mesalamine) and a sulfapyridine moiety. It is therefore used for colonic disease, either as initial therapy or to maintain remission. Adverse effects are mainly caused by the sulfapyridine moiety and include headache, vomiting, and abdominal pain. A reduction in dose is usually beneficial. Newer 5-ASA preparations lack the sulfa moiety of sulfasalazine and are associated with fewer side effects. Mesalamines are slow-release formulae of 5-ASA and are effective as a primary tool for initial and maintenance therapy of IBD. Rare hypertensitivity reactions occur and include pneumonitis, pancreatitis, and hepatitis. Recently, several case reports have been published suggesting an association between the use of 5-ASA and the development of chronic tubulointerstitial nephritis in patients with IBD $[4,5]$. Because of adverse effects of these agents, differentiation of renal complications subtending these therapies from the true extraintestinal manifestations of IBD involves much difficulty.

In this review, we note the drugs including 5-ASA associated nephrotoxicity and also show case reports of UC related nephritis.

\section{Drugs of the treatment for IBD associated nephrotoxicity}

\subsection{Epidemiology of nephrotoxicity in IBD}

Sulfasalazine has been used in the treatment of IBD, both for UC and for Crohn's disease. Newer 5-ASA preparations lack the sulfa moiety of sulfasalazine and are associated with fewer side effects. Mesalamines are slow-release formulae of 5-ASA and are effective as a primary tool for initial and maintenance therapy of IBD. Azad Khan et al. studied the therapeutic activity of the component parts of sulfasalazine and found that 5-ASA was the 
therapeutically active component of the drug [6]. In moderate active UC, both sulfasalazine and 5-ASA have proven to be effective in inducing and maintaining clinical remission. However, a number of cases have shown the 5-ASA related toxicity [7, 8]. In particular, nephrotoxicity has been described in some patients with IBD treated with 5-ASA [7, 8]. In this respect, both acetylsalicylic acid and phenacetin, which have been implicated in the occurrence of nonsteroidal antiinflammatory drug-induced nephropathy, share structural similarities with 5-ASA [9, 10]. Furthermore, previous studies reported that 5-ASA may cause injuries to tubular epithelial cells in animals when fed in high doses [9, 10]. The actual incidence of nephrotoxicity in IBD patients with 5-ASA therapy has not been determined, but it has been suggested that renal impairment may occur in up to $1 \%$ of patients treated with 5-ASA. A recent prospective study revealed that renal impairment was observed in 2$3 \%$ of IBD patients with and without concomitant 5-ASA treatment [11]. More recently, a case-control analysis found that IBD patients treated with 5-ASA had an increased risk of renal disease [12]. However, after adjustment for several factors and variables, the risk of 5ASA users was comparable to controls. This study found that IBD patients without 5-ASA also had increased risk of renal disease. Taken together, although users of 5-ASA may have an increased risk of renal disease, it may be partly attributable to the underlying disease [12].

\subsection{Monitoring markers in IBD}

Microalbuminuria has been demonstrated to be present in the majority of IBD patients, and it seems to be related to disease activity. However, other studies have shown that microalbuminuria is not present in patients with IBD [13]. Some authors have concluded that an increased prevalence of tubular proteinuria may be attributed to high doses of 5ASA [14]. Nevertheless, differences among these studies may be related to differences in disease activity of IBD. Taken together, it is important to conduct a systematic evaluation of the effect of 5-ASA treatment on renal function in patients with IBD.

5-ASA treatment-related nephrotoxicity is reported most often within the first 12 months, but also delayed presentation after several years has been observed. Thus, regular monitoring of renal function should be performed during the therapy.

Several attempts have been made to measure early signs of renal impairment in patients with IBD treated with 5-ASA using sensitive markers of glomerular and tubular dysfunction. Riley et al. found that the incidence of elevated urinary markers such as Nacetyl-D-glucosamidase is low in patients with quiescent UC, which is independent of the dose and duration of 5-ASA treatment [15]. When renal damage occurs, its presence is unlikely to be detected by urinalysis in its early remediable stages. Although tubular enzymuria may be a more sensitive and specific marker of renal damage, it is not yet available as a screening method and the correlation between the several urinary markers of renal damage and 5-ASA treatment remains unproven. These limitations emphasize the importance of monitoring serum creatinine in patients with IBD treated with 5-ASA.

\subsection{The incidence of renal disease in IBD}

It has been suggested that mesalazine may induce renal impairment more frequently than sulfasalazine [16]. In an analysis of spontaneous reports of adverse events in the UK, 5-ASArelated nephrotoxicity seemed more frequent in mesalazine-treated patients compared with 
sulfasalazine-treated patients [17]. Recently, data from the UK General Practice Research Database were used to estimate the incidence of renal disease in adult patients with IBD, and mesalazine and sulfasalazine users had comparable risks of nephrotoxicity ( 0.17 versus 0.29 cases per 100 person-years, respectively) [12]. It can be conluded that the nephrotoxicity potential of mesalazine and sulfasalazine seems to be similar, and,even if differences exist, they are probably small. Mesalazine should be withdrawn when renal impairment manifests in a patient with IBD in whom no other cause can be readily identified. If withdrawal of 5ASA treatment does not result in a fall in serum creatinine, then the patient should be referred for consideration of renal biopsy to make sure whether interstitial nephritis or glomerulonephritis associated with IBD is the cause of the persistent impaired renal function.

\subsection{Treatment for renal impairment in IBD}

Steroids and azathioprine have been used in patients with renal impairment due to mesalazine-associated interstitial nephritis, but the evidence for beneficial roles is anecdotal and uncontrolled. Partial improvement or even complete recovery of renal function after steroid therapy has been reported by several authors. However, other studies have been unable to demonstrate a beneficial effect of these immunosuppressive drugs. Nevertheless, it has been suggested that a trial of high-dose steroid $(60 \mathrm{mg} /$ day or $1 \mathrm{mg}$ day $/ \mathrm{kg}$ for up to 3 months) may be recommended in patients whose renal function does not respond to drug withdrawal alone [7]. Although most case reports indicate reversibility after cessation of the drug, in some cases permanent clinical kidney dysfunction has been observed. Thus, it has been calculated that $10 \%$ of the patients with 5-ASA nephrotoxicity will develop end-stage renal disease [5].

\section{Case reports of UC related nephritis}

\subsection{ANCA related nephritis}

UC is typically associated with antineutrophil cytoplasmic antibodies of perinuclear type (pANCA). These antibodies are not usually considered to carry potential for the development of systemic vasculitis as they lack specificity for proteinase 3 (PR3) or myeloperoxidase (MPO). ANCA can be detected in sera from patients with a wide variety of inflammatory diseases including UC. In one study of 50 patients with UC, $54 \%$ were shown to be either pANCA or c-ANCA positive but none of these antibodies reacted with PR3 or MPO [18]. In another study, ANCA-positive patients with UC were followed for a year during which no evidence of glomerulonephritis was found [19].

\section{$3.2 \lg$ A nephropathy}

UC may be associated with a number of extraintestinal complications, involving almost any organ system. The organs most commonly involved include the skin, joints, biliary tract and eyes $[3,20]$. However, renal and genitourinary tract manifestations are quite rare, particularly glomerulonephritis. They reported that a patient of $\operatorname{IgA}$ nephropathy with UC and chronic intermittent episodes of indolent macrohematuria [21]. IgA nephropathy can be primary in most cases or secondary but is rarely associated with UC $[22,23]$. Altered T-helper cells' function might be the initial common derangement of both UC and IgA nephropathy [24, 25]. In IgA nephropathy such alteration in CD4-positive T 
cells causes a nonspecific stimulus on plasma cells in the bone marrow to secrete polymeric IgA1 into the circulation [26, 27] and of IgG1 and IgG3 in UC [28, 29] culminating in both cases with a common state of local cytokine secretion and tissue inflammation.

\subsection{UC related interstitial nephritis}

Lately, we reported a patient with UC who has developed acute interstitial nephritis and the subsequent renal failure following a long pause of the treatment with mesalazine [30]. In this case, we observed progressive decline in renal function in a patient with UC. Although the patient exhibited stable levels of serum $\mathrm{Cr}$ during the 3 year period after the treatment with mesalazine and sulfapyridine was discontinued, he developed severe interstitial nephritis associated with moderately active UC (Figure 1). His renal biopsy samples showed evidence of severe active tubulointerstitial nephritis along with intense renal interstitial infiltration of CD3-positive T cells (Figure 2). Colonic fiberscopic examination also revealed moderate UC activity and the mucosal infiltration of CD3-positive cells, thus suggesting the common immune mechanism possibly mediated by T-cell dysregulation. Since the patient had not used any nephrotoxic agent for at least three years, it was reasonable to conclude

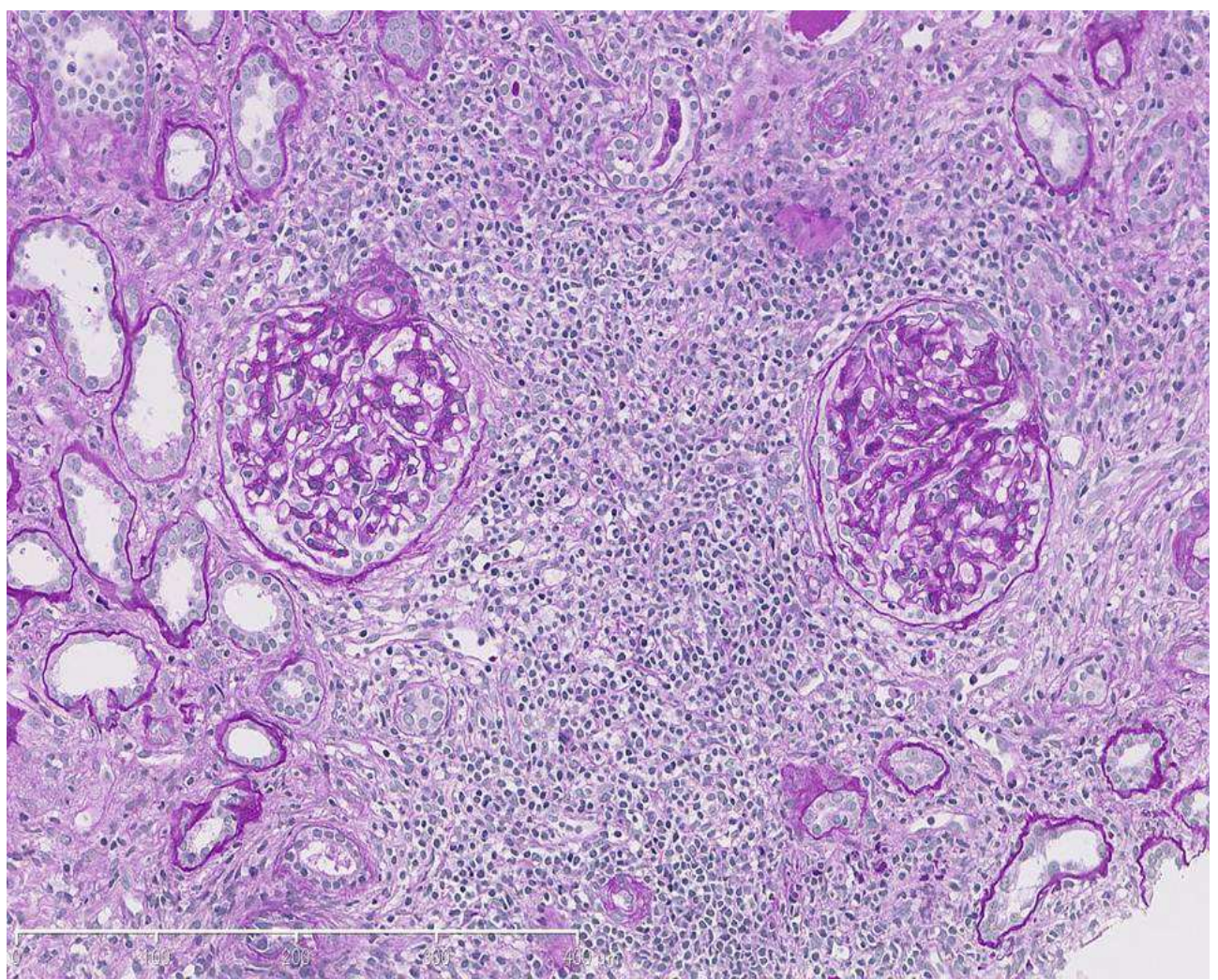

Fig. 1. UC related interstitial nephritis (PAS staining) 


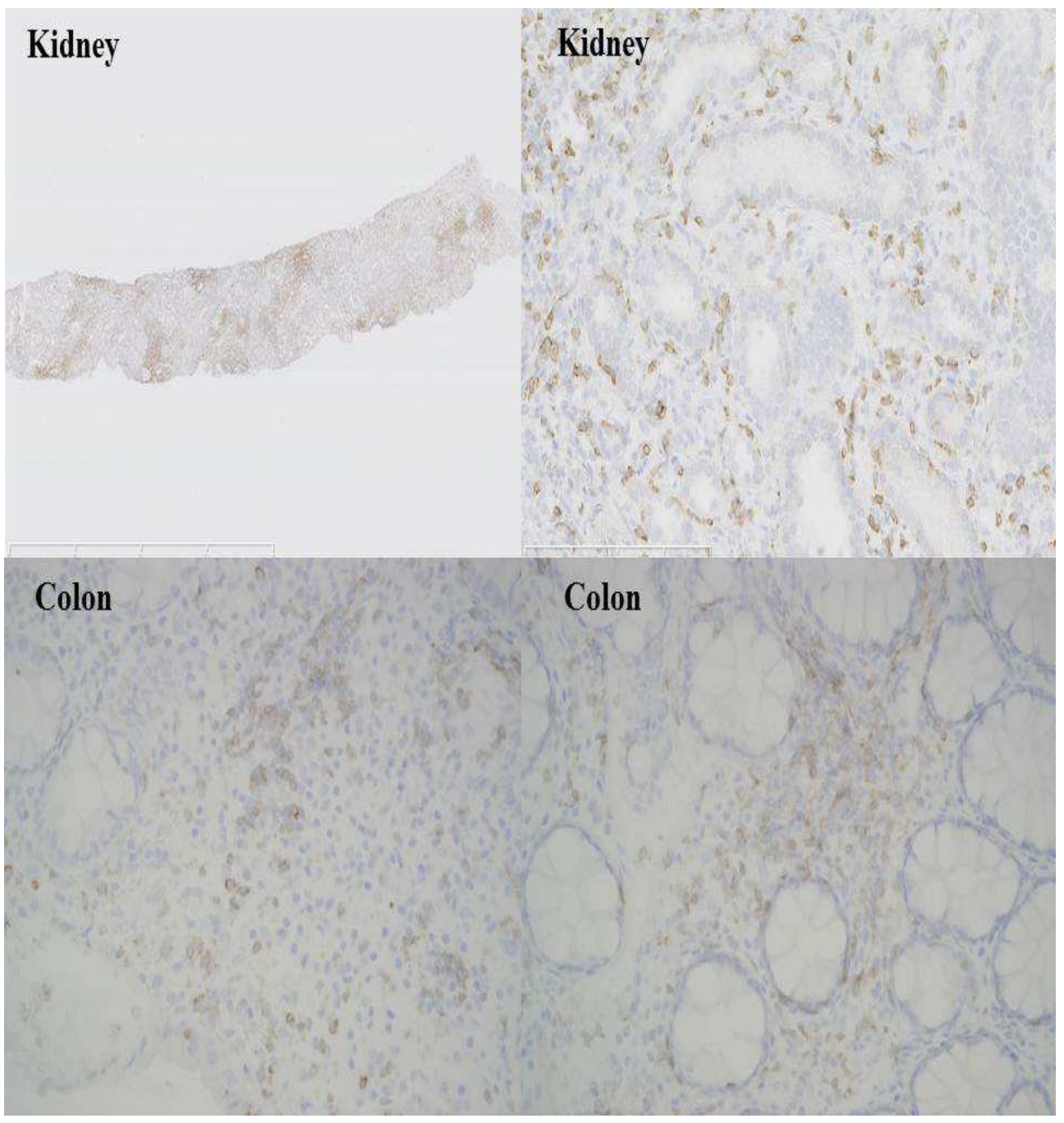

Fig. 2. Intense interstitial infiltration of CD3-positive T cells was detected in the kidney (Upper). The infiltration of CD3-positive cells into the intestinal mucosa was also observed (Lower)

that the main precipitating cause of the progression of renal injury during the medicationfree period is attributable to the disease activity of UC per se, rather than the flare-up of the reminiscence of mesalazine effect.

Drug-induced nephropathy constitutes a critical problem that precludes the continued use of the agent. Nephrotoxicity has been described in patients with IBD treated with 5-ASA [4, 5]. In the literature survey, 5-ASA-associated nephrotoxicity is reported most often within the first 12 months from the initiation of the drug [31], but delayed presentation has also been shown rarely, with the onset of the renal manifestation after several years of the 
treatment $[32,33]$. In most of their reports, however, 5-ASA was given continuously during the latent period. In our case, by contrast, nephrotoxic agents, including mesalazine or sulfapyridine, were discontinued for at least three years, during which renal function remained relatively stable. Collectively, it appears unlikely that the aggravating process after the cessation of the drugs is associated with the direct nephrotoxic effect of these agents.

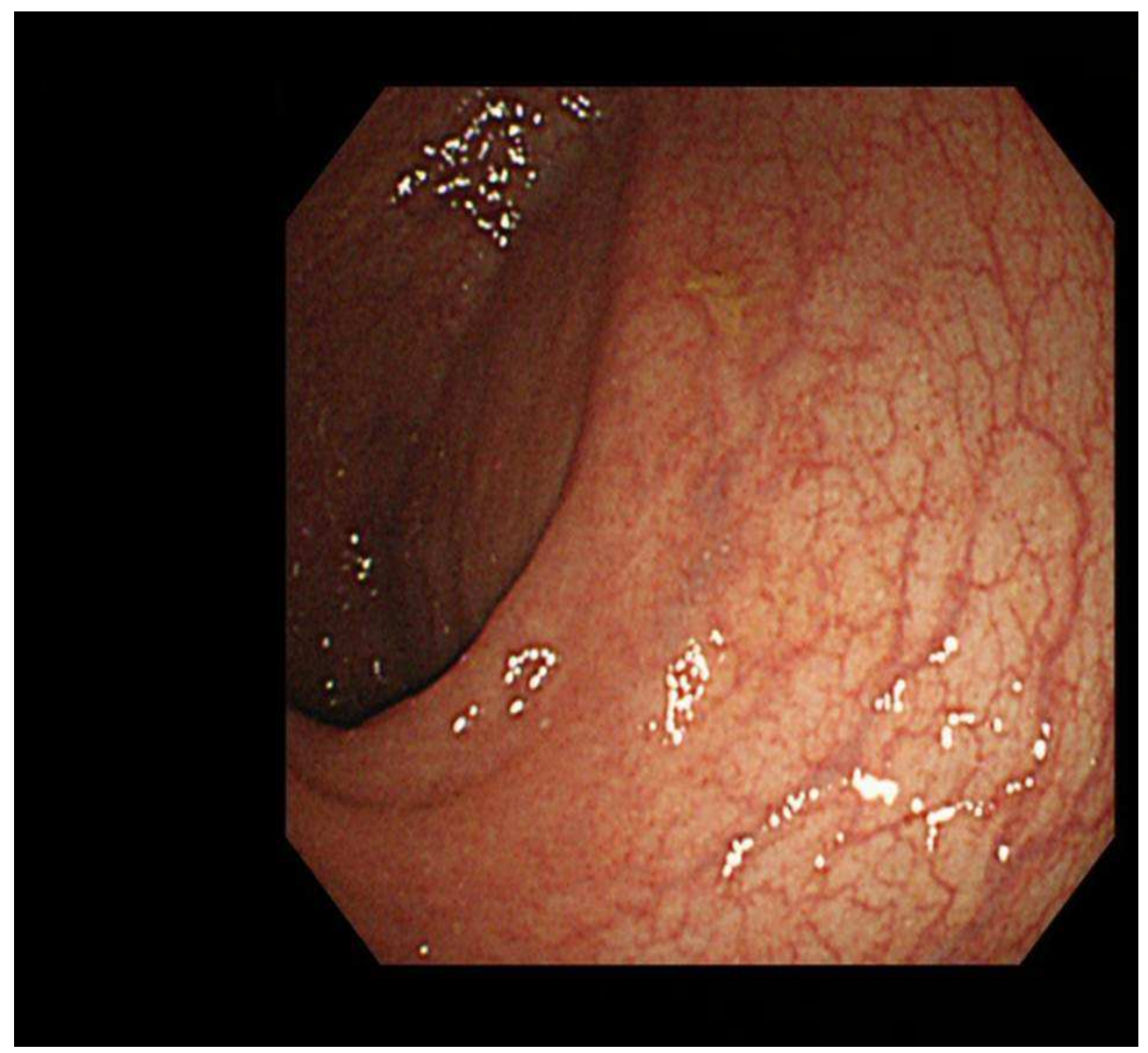

Fig. 3. Colonic fiberscopic findings unveiled moderate UC activity

Since IBD is acknowledged as autoimmune disease affecting multiple extraintestinal organs, it is possible that the kidney is a target organ for the UC-associated systemic injury. Indeed, several types of kidney disease have been documented, including glomerulonephritis, membranous nephropathy and nephrotic syndrome as rare extraintestinal manifestations of IBD [34-35]. In contrast to glomerular disease, tubulointerstitial nephritis unrelated to nephrotoxic agents has rarely been reported hitherto [36]. Of note, it has been shown that a substantial number of patients manifest 
pathological enzymuria [37]. Furthermore, a strong correlation between disease activity and tubular proteinuria has been reported in IBD [38]. In our recent case report, the patient shows moderate UC activity (Figure 3) and progressive course of interstitial nephritis with no nephrotoxic agent given during the antecedent 3-year period. Although there reported one case showing that the renal injury does not parallel the activity of IBD [33], the absence of other aggravating factors rather favors the recognition of the UC activity as a precipitating mechanism in this case.

Link between IBD and kidney disease merits comment. As shown in the present case, the kidney constitutes a target organ involved in the UC-induced systemic disorders. Furthermore, the kidney, where various drugs and their metabolites are condensed in situ and excreted in the urine, is susceptible to the nephrotoxicity of these agents. Of more clinical importance, the present case sheds light on the kidney as an organ affected in IBD albeit low incidence reported so far. To the extent that the kidney disease contributes substantially to the development of cardiovascular events, our observation would emphasize the need for increasing awareness of the kidney in the management of IBD.

\section{Conclusion}

A large number of biological agents as well as many biochemical substances and molecules specifically for the medical treatment of patients with IBD, have been developed. Sulfasalazine has been used in the treatment of IBD, both for UC and for Crohn's disease. Mesalamines are slow-release formulae of 5-ASA and are effective as a primary tool for initial and maintenance therapy of IBD. Recently, several case reports have been published suggesting an association between the use of 5-ASA and the development of chronic tubulointerstitial nephritis in patients with IBD. Because of adverse effects of these agents, differentiation of renal complications subtending these therapies from the true extraintestinal manifestations of IBD involves much difficulty. Since IBD is acknowledged as autoimmune disease affecting multiple extraintestinal organs, it is possible that the kidney is a target organ for the UC-associated systemic injury. Indeed, several types of kidney disease have been documented, including glomerulonephritis, membranous nephropathy and nephrotic syndrome as rare extra-intestinal manifestations of IBD. We also reported the case of acute interstitial nephritis associated with UC. As noted in our case report, we assume that the renal manifestation is attributed to intrinsic disease process of the UC-mediated immune dysregulation, and emphasize the need for the pathophysiological evaluation of the intestine and other organs in clinical situations.

\section{References}

[1] Podolsky DK. (2002). Inflammatory bowel disease. N Engl J Med, Vol 347, No. 6, (August 2002), pp. 417-429.

[2] Ricart E. (2004). Autoimmune disorders and extraintestinal manifestations in first-degree familial and sporadic inflammatory bowel disease: a case-control study. Inflamm Bowel Dis, Vol 10, No. 3, (May 2004), pp. 207-214. 
[3] Das KM. (1999). Relationship of extraintestinal involvements in inflammatory bowel disease: new insights into autoimmune pathogenesis. Dig Dis Sci. Vol 44, pp. 113.

[4] Uslu N (2007). Acute tubular injury associated with mesalazine therapy in an adolescent girl with inflammatory bowel disease. Dig Dis Sci. Vol 52, pp. 2926-2929.

[5] Arend LJ (2004). Interstitial nephritis from mesalazine: case report and literature review. Pediatr Nephrol. Vol 19, No. 5, (May 2004), pp. :550-553.

[6] Azad Khan AK (1977). An experiment to determine the active therapeutic moiety of sulphasalazine. Lancet.Vol 29, No. 2, (October 1977), pp. 892-895.

[7] Corrigan G (2000). Review article: interstitial nephritis associated with the use of mesalazine in inflammatory bowel disease. Aliment Pharmacol Ther. Vol. 14, No. 1, (January 2000), pp. 1- 6.

[8] Loftus EV (2004). Systematic review: short-term adverse effects of 5-aminosalicylic acid agents in the treatment of ulcerative colitis. Aliment Pharmacol Ther. Vol.19, No. 2, (January 2004), pp. 179 -189.

[9] Calder IC (1972). Nephrotoxic lesions from 5-aminosalicylic acid. BMJ.Vol. 15, No. 1, (Jan 1972), pp. 52-54.

[10] Bilyard KG (1990). Mesalazine: an overview of key preclinical studies. Scand J Gastroenterol Suppl. Vol. 172, pp. 52-55.

[11] Elseviers MM (2004). Renal impairment in patients with inflammatory bowel disease: association with aminosalicylate therapy? Clin Nephrol. Vol. 61, No. 2, (February 2004), pp. 83- 89.

[12] Van Staa TP (2004). 5-Aminosalicylic acids and the risk of renal disease: a large British epidemiologic study.Gastroenterology. Vol. 126, No. 7, (June 2004), pp.1733-1739.

[13] Fraser JS (2001). Renal tubular injury is present in acute inflammatory bowel disease prior to the introduction of drug therapy. Aliment Pharmacol Ther. Vol.15, No. 8, (August 2001), pp. 1131-1137.

[14] Schreiber S (1997). Renal tubular dysfunction in patients with inflammatory bowel disease treated with aminosalicylate. Gut. Vol. 40, No. 6, (June 1997), pp. 761766.

[15] Riley SA (1992). Tests of renal function in patients with quiescent colitis: effects of drug treatment. Gut. Vol. 33, No. 10, (October 1992), pp. 1348-1352.

[16] Birketvedt GS (2000). Glomerular and tubular renal functions after long-term medication of sulphasalazine, olsalazine, and mesalazine in patients with ulcerative colitis. Inflamm Bowel Dis. Vol. 6, No. 4, (November 2000), pp. 275-279.

[17] Ransford RA (2002). Sulphasalazine and mesalazine: serious adverse reactions reevaluated on the basis of suspected adverse reaction reports to the Committee on Safety of Medicines. Gut. Vol. 51, No. 4, (October 2002), pp. 536-539.

[18] Cambridge G (1992). Antineutrophil antibodies in inflammatory bowel disease: prevalance and diagnostic role. Gut. Vol. 33, No. 5, (May 1992), pp. 668-674.

[19] Rosa M (1996). Does the presence of ANCA in patients with ulcerative colitis necessarily imply renal involvement? Nephrol Dial Transplant. Vol.11, No. 12, (December 1996), pp. 2426-2429.

[20] Monsen U (1990). Extracolonic diagnoses in ulcerative colitis: An epidemiological study. Am J Gastroenterol. Vol. 85, No. 6, (June 1990), pp. 711-716. 
[21] Trimarchia HM (2001). Immunoglobulin A Nephropathy and Ulcerative Colitis. Am J Nephrol. Vol. 21, No. 5, (September 2001), pp. 400-405.

[22] Dard S (1983). A new association: Ankylosing spondylitis and Berger's disease. Kidney Int. Vol. 24, (August 1983), pp129.

[23] Bruneau C (1986). Seronegative spondyloarthropathies and IgA glomerulonephritis: A report of four cases and a review of the literature. Semin Arthritis Rheum Vol. 15, No. 3. (February 1986), pp. 179-184.

[24] Imasawa T (1999). Bone marrow transplantation attenuates murine IgA nephropathy: Role of a stem cell disorder. Kidney Int. Vol. 56, No. 5, (November 1999), pp. 18091817.

[25] Noris M (1999). IgA nephropathy: A stem cell disease? Kidney Int. Vol. 56, No. 5, (November 1999), pp. 1964-1966.

[26] Van den Wall Bake A (1989). Elevated production of polymeric and monomeric IgA1 by the bone marrow in IgA nephropathy. Kidney Int . Vol. 35, No. 6, (June 1989), pp. 1400-1404.

[27] Van Es L (1992). Pathogenesis of IgA nephropathy. Kidney Int. Vol. 41, No. 6, (June 1992), pp. 1720-1729.

[28] Scott M (1986). Spontaneous secretion of IgG subclasses by intestinal mononuclear cells: Differences between ulcerative colitis, Crohn's disease and control subjects. Clin Exp Immunol. Vol. 66, No. 1, (October 1986), pp. 209-216.

[29] Kett K (1987). Mucosal subclass distribution of immunoglobulin G-producing cells is different in ulcerative colitis and Crohn's disease of the colon. Gastroenterology. Vol. 93, pp. 919-924.

[30] Tokuyama H (2010). Acute interstitial nephritis associated with ulcerative colitis. Clin Exp Nephrol. Vol.14, No. 5, (October 2010), pp. 483-6.

[31] Popoola J (1998). Late onset interstitial nephritis associated with mesalazine treatment. Brit Med J. Vol. 317, (September 1998), pp. 795-797.

[32] Gisbert JP (2007). 5-Aminosalicylates and renal function in inflammatory bowel disease: a systematic review. Inflamm Bowel Dis. Vol. 13, No. 5, (May 2007), pp. 629-638.

[33] Wilcox GM (1990). Glomerulonephritis associated with inflammatory bowel disease. Report of a patient with chronic ulcerative colitis, sclerosing cholangitis, and acute glomerulonephritis. Gastroenterology. Vol. 98, No. 3, (March 1990), pp. 786791.

[34] Ridder RM (2005). Membranous nephropathy associated with familial chronic ulcerative colitis in a 12-year-old girl. Pediatr Nephrol. Vol. 20, No. 9, (September 2005), pp. 1349-1351.

[35] Nand N (1991). Nephrotic syndrome in ulcerative colitis. Nephrol Dial Transplant. Vol.6, No. 3, pp.227.

[36] Khosroshahi HT (2006). Tubulointerstitial disease and ulcerative colitis. Nephrol Dial Transplant. Vol. 21, No. 8, (August 2006), pp. 2340.

[37] Kreisek W (1996). Renal tubular damage: an extraintestinal manifestation of chronic inflammatory bowel disease. Eur J Gastroenterol Hepatol. Vol. 8, No. 5, (May 1996), pp. 461-468. 
[38] Herrlinger KR (2001). Minimal renal dysfunction in inflammatory bowel disease is related to disease activity but not to 5-ASA use. Aliment Pharmacol Ther. Vol.15, No. 3, (March 2001), pp.:363-369. 


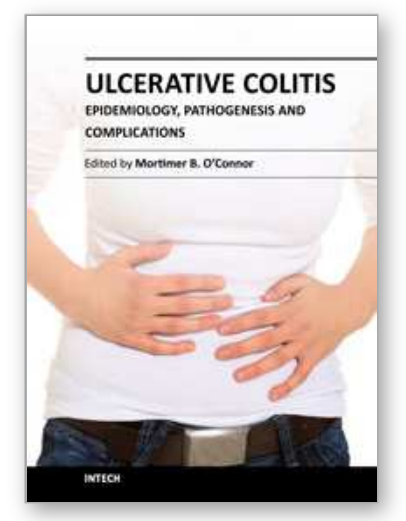

\author{
Ulcerative Colitis - Epidemiology, Pathogenesis and Complications \\ Edited by Dr Mortimer O'Connor
}

ISBN 978-953-307-880-9

Hard cover, 280 pages

Publisher InTech

Published online 14, December, 2011

Published in print edition December, 2011

This book is intended to act as an up-to-date reference point and knowledge developer for all readers interested in the area of gastroenterology and in particular, Ulcerative Colitis. All authors of the chapters are experts in their fields of publication, and deserve individual credit and praise for their contributions to the world of Ulcerative Colitis. We hope that you will find this publication informative, stimulating, and a reference point for the area of Ulcerative colitis as we move forward in our understanding of the field of medicine.

\title{
How to reference
}

In order to correctly reference this scholarly work, feel free to copy and paste the following:

Hirobumi Tokuyama, Shu Wakino, Koichi Hayashi and Hiroshi Itoh (2011). Nephritis Associated with Ulcerative Colitis, Ulcerative Colitis - Epidemiology, Pathogenesis and Complications, Dr Mortimer O'Connor (Ed.), ISBN: 978-953-307-880-9, InTech, Available from: http://www.intechopen.com/books/ulcerative-colitis-epidemiologypathogenesis-and-complications/nephritis-associated-with-ulcerative-colitis

\section{INTECH}

open science | open minds

\section{InTech Europe}

University Campus STeP Ri

Slavka Krautzeka 83/A

51000 Rijeka, Croatia

Phone: +385 (51) 770447

Fax: +385 (51) 686166

www.intechopen.com

\section{InTech China}

Unit 405, Office Block, Hotel Equatorial Shanghai

No.65, Yan An Road (West), Shanghai, 200040, China

中国上海市延安西路65号上海国际贵都大饭店办公楼 405 单元

Phone: +86-21-62489820

Fax: $+86-21-62489821$ 
(C) 2011 The Author(s). Licensee IntechOpen. This is an open access article distributed under the terms of the Creative Commons Attribution 3.0 License, which permits unrestricted use, distribution, and reproduction in any medium, provided the original work is properly cited. 